\title{
A Phase Cell Approach to Yang-Mills Theory
}

\author{
IV. The Choice of Variables*
}

Paul Federbush

Department of Mathematics, University of Michigan, Ann Arbor, MI 48109, USA

\begin{abstract}
Variables are chosen to describe the continuum Yang-Mills fields, a discrete set of group valued variables. These are group elements associated to the sequence of lattice field theory configurations realizing the continuum field. The field is "laid down" inductively. At each inductive step one of three types of "field excitations" makes its contribution to the total field. These are either "pure modes", "averaging correction modes", or "chunks". The pure modes are small field excitations, as studied in previous papers in this series $[2,3]$. The averaging correction modes are small excitations added to make sure the block spin transformation is satisfied at each edge. The chunks, encompassing most of our difficulties, are large field excitations. Topological obstructions in $\pi_{3}(G)$ must be dealt with in defining a gauge choice for each chunk. The laying down process is complex, but fiendishly clever, ensuring a principle of "gauge invariant coupling". Each group valued variable is either the "amplitude" of a pure mode or an "internal variable" in a chunk: The amplitude of an averaging correction mode is a dependent variable, a function of the (independent) variables used to describe the field. The (independent) variables herein defined are those whose mutual interaction will later be inductively decoupled in defining the phase cell cluster expansion (of course treating the variables of each chunk as a unit).
\end{abstract}

\section{Introduction}

Loosely speaking one may separate the work of controlling a cluster expansion into two tasks. The first, the "non-linear" aspect, is controlling the functional integrals using positivity properties of the action. The second, the "linear" aspect, is

\footnotetext{
* This work was supported in part by the National Science Foundation under Grant No. PHY-
} 85-02074 
exhibiting the renormalization cancellations. Of course one cannot totally separate these two aspects. In the phase cell cluster expansion, however, the separation is rather complete. The renormalization cancellations in our theory are basically no different from those in a super-renormalizable theory; there are only a finite number of divergent structures to deal with. Our choice of variables has in mind facilitating the treatment of both the linear and non-linear developments to follow (in the remaining papers in this series).

Our treatment of the four dimensional Yang-Mills theory will utilize four basic ingredients, the real ideas that make our cluster expansion work. (We are speaking of ideas beyond the general idea of studying the lattice gauge theory via the renormalization group, a program initiated by Balaban:)

1) The block spin transformation of Bałaban in the small field region [1, Eq. (1:8)].

2) The modified block spin transformation (agreeing with 1) in the small field region) for general field configurations [4].

3) The choice of variables, as defined in the present paper. This involves describing the three kinds of (field) excitations, and detailing their incorporation into the total field.

4) The interpolation scheme for defining the "partial actions". This will be developed in Paper VII in this series. It is our contention that with these ideas our cluster expansion, though horrendously complicated, will be straightforward.

In defining the "field excitations" we incorporate as a physical principle the idea that a field excitation of a given scale is defined in the background field of the excitations at larger scales. There is a second dominating principle, that the excitation couples to the larger scale excitations through the $F$ field, rather than the $A$ field, of the large scale excitations. We further describe this latter principle (which we will call gauge invariant coupling). In the case that the large scale excitations provide a pure gauge transformation (the corresponding $g_{o p}=I d$ ) there is no coupling to the lower scale excitations. (One in fact must make simple translations in the lower scale variables to exhibit this.) In general a term coupling an excitation at length scale $L$, to an excitation at a smaller length scale $\ell$, has associated to it a numerical factor $(\ell / L)^{2}$ in the interaction. This is crucial for renormalization cancellations, to enable the perturbation theory aspects of the expansion to be handled.

Loosely speaking, when one adds an excitation living near a point $x_{0}$ to the field (of larger scale excitations) already present, one first performs a gauge transformation of this background field to a radial axial gauge radiating out from $x_{0}$. One then superimposes the new excitation by adding $A$ fields. Thereafter the gauge transformation is undone. The $A$ fields are as small as possible near $x_{0}$ for given $F$ fields, in the radial gauge. This definition of superposition yields final $\left|g_{\partial p}\right|$ values (essentially) independent of the gauge in which the background field appears (see Sect. 7).

We herein enter a casual digression on the renormalization cancellations (not to be explicitly treated until several papers down this series). In phase cell expansions such as used by the author or Magnen and Sénéor - one never sees vacuum diagrams. I will discuss briefly those "structures" that do require cancellation in a phase cell procedure. 


$$
\begin{aligned}
& \text { level } n=0 \\
& \text { level } \begin{aligned}
n & =n \\
L & =\varepsilon=1 / N^{n}
\end{aligned}
\end{aligned}
$$

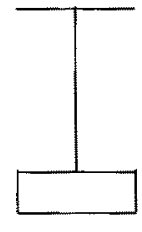

(a)

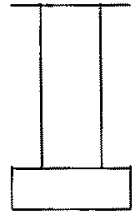

(b)

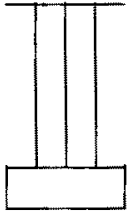

(c)

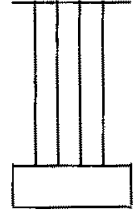

(d)

In the figures we visualize terms arising by trying to decouple a variable at level 0 , that introduces variables in the lattice of edge size $\varepsilon$. The figures represent decoupled terms. The four figures distinguish situations involving 1st, $2 \mathrm{nd}, 3 \mathrm{rd}, 4$ th order polynomials in the large scale variable (higher order powers are harmless). Long experience shows these are the crucial terms to understand and handle, if a procedure treats these successfully it will work. These structures are now briefly analyzed in three models.

a) $\phi_{4}^{4}$ with wrong sign of $\lambda$ : Figures (a) and (c) will not appear if the theory is invariant under $\phi \rightarrow-\phi$. Terms in (b) have associated numerical factors $\sim \varepsilon^{2}$ and need factors $\sim \varepsilon^{4}$ for localization. (This is the quadratic divergence $q^{2} \sim 1 / \varepsilon^{2}$.) These terms must be cancelled by renormalization counterterms - there are an infinite number of them, so the cancellation must be non-perturbative: Magnen and Sénéor have accomplished this. For Yang-Mills there will be no necessary non-perturbative cancellations! $s^{\text {th }}$ order terms in (d) have associated numerical factors $\varepsilon^{4}\left(\frac{1}{\ln \varepsilon^{-1}}\right)^{a s}$ and require $\varepsilon^{4}$ for localization. If $s>1 / a$ one has enough numerical factors to control the sum over values of $n$, the terms with $s \leqq 1 / a$ must be cancelled. Thus in (d) there are only a finite number of terms, logarithmically divergent, that must be cancelled!

b) $2-d$ non-linear $\sigma$ model: Terms in (a) and (c) will not appear. Terms in (d) are harmless. Terms in (b) of order $s$ have associated numerical factors $\varepsilon^{2}\left(\frac{1}{\ln \varepsilon^{-1}}\right)^{a s}$ and require $\varepsilon^{2}$ for localization. The situation is as in (d) above.

c) 4-d Yang-Mills: Terms in (c) and (d) are harmless. Terms in (a) do not appear by a gauge invariance type argument. Terms in (b) are exactly as terms in (d) of the $\phi_{4}^{4}$ situation. [Requiring $\varepsilon^{4}$, having associated factors $\varepsilon^{4}\left(1 / \ln \varepsilon^{-1}\right)^{a s}$.]

It is important to emphasize these estimates are obtained using smallness factors that arise from the gauge invariant coupling procedure: [If one allowed coupling to $A$ not $F$ then a numerical factor of $\ell / L$ instead of $(\ell / L)^{2}$ would arise, a disaster.] This ends our digression on renormalization cancellations.

If one fixes the excitations, variables, down to some scale, then the lower scale excitations can be visualized as excitations of the Yang-Mills field about the configuration that minimizes the action subject to the constraint of having the larger scale excitations fixed in some configuration. With our definition of the excitations, the expansion is not about the exact minimum configuration, but is about a point close enough to the minimum for purposes of the expansion.

The "small field modes" of [2,3] are the starting point for defining our two types of mode excitations. These make their contribution to the total field in a manner chosen to meet the gauge invariant coupling principle. The total field configuration is 
laid down inductively one excitation at a time. The first excitation specifies group elements for all bonds at all scales; the second excitation modifies all these assignments, etc. The assignments do not automatically satisfy the averaging requirement (that the assignments at one scale determine by the block spin transformation the assignments at the next larger scale) and averaging corrections are also made inductively (by laying down averaging correction modes). In the end the field configuration assigns a group element to a bond at a given scale, that depends on the variables, excitations, of that scale and all larger scales in a very complicated manner.

In [4] a parameter, $a$, is introduced defining the division between small and large plaquette field. We introduce a $C^{\infty}$ partition of unity of $[0, \infty)$,

where

$$
1=\chi_{s}(x)+\chi_{L}(x)
$$

$$
\begin{array}{ll}
\chi_{s}(x)=0, & x>a, \\
\chi_{L}(x)=0, & x<a / 2 .
\end{array}
$$

We introduce into the functional integration measure $\int d \mathscr{F}$ a product

$$
\prod_{p}\left(\chi_{S}\left(\left|g_{\partial p}\right|\right)+\chi_{L}\left(\left|g_{\partial p}\right|\right)\right)
$$

Selecting a $\chi_{S}$ or a $\chi_{L}$ for each plaquette we get a $S-L$ configuration, $c$. We write

$$
\int d \mathscr{F}=\sum_{c \in \mathscr{B}} \int d \mathscr{F}_{c} .
$$

$\mathscr{C}$ is the set of all such configurations. The sum in (0.4) is analogous to a Peierls expansion (as appears in treatments of Debye screening). The $S-L$ configuration in which all plaquettes are $S$-plaquettes (there are no L-plaquettes) is called the pure small field configuration. In this situation there are a collection of edges, $\mathscr{E}_{p}$, defined below, to each of which a pure mode is associated. There are a collection of edges, $\mathscr{E}_{A}$, defined below, to each of which an averaging correction mode is associated. The total field is built up of these mode contributions.

In a general $S-L$ configuration, some of the pure modes associated to edges "close" to $L$-plaquettes, are not used in constructing the total field. Their contribution is replaced by contributions of chunks. Each chunk, in some loose sense, provides the field contribution of some of the displaced pure modes, in a situation where these displaced modes may have large amplitudes. The total field is inductively built up of chunk contributions, the remaining pure mode contributions, and all the averaging correction mode contributions.

Each mode and each chunk considered in isolation yields assignments to all bonds at all levels, its "isolated" field. For pure modes, and chunks, these isolated assignments satisfy averaging (are consistent with the block spin transformations), and thus arise from a continuum field, their isolated continuum field contribution. (Remember the duality between continuum fields and compatible assignments to the lattice fields $[2,5]$.) An averaging correction mode's isolated field does not satisfy averaging, at a single edge (and does satisfy averaging at all other edges). It still has an associated continuum field contribution to which the lattice fields converge. 
The total field is assembled by laying down the (isolated field) contributions of modes and chunks inductively. We use $\mathscr{C}_{i}$ to denote the isolated field of excitation $i$. In the induction procedure, if the field before excitation $i$ is laid down is $\mathscr{F}_{i-1}$, then the resulting field after $\mathscr{C}_{i}$ is laid down is denoted by

$$
\mathscr{C}_{i} \times \mathscr{F}_{i-1}
$$

When the first $n$ excitations have been laid down the field is

$$
\mathscr{F}_{n}=\mathscr{C}_{n} \times \mathscr{C}_{n-1} \times \ldots \times \mathscr{C}_{1} .
$$

The "twisted products" defining the laying down process will be defined later.

The isolated fields of modes will be easily extracted from the analysis in $[2,3]$, yielding all necessary estimates. The brunt of our efforts in this paper will be to define and estimate the isolated fields of chunks, an effort continuing into the next paper in this series. The touchiest task is the definition of a gauge for the isolated chunk field. Construction of a gauge involves us with topological obstructions, in $\pi_{3}(G)$ [we have assumed $\pi_{1}(G)=0$, and $\pi_{2}(G)=0$ for free], obstructions that would not appear in three dimensions. Our choice of a gauge is one that has possible isolated point singilarities.

We recall that gauge fields have long been called an "essentially non-linear field theory". Our complicated expression for the Yang-Mills field, a "twisted product" of contributions from modes (and chunks), may be viewed as the non-linear counterpart of the expansion $\phi(x)=\sum \alpha_{k} u_{k}(x)$ for a scalar field. In the pure small field configuration, if $G$ were commutative, these expansions would be quite the same. The scalar field is linearly related to its discrete set of real number valued variables, $\alpha_{k}$; the Yang-Mills field is a complicated, non-linear, function of its discrete set of group valued variables (herein defined). We suggest that for those whose technique is to integrate out variables level by level, such as Bataban, Benfatto, Gallavotti et al., Gawedzki and Kupiainen, the formalism of the present paper may provide a good choice of variables to integrate out - in particular a good gauge.

In the body of the paper we will often use the abbreviations $P$-modes and $A$ modes to denote pure modes and averaging correction modes.

\section{Preliminaries, Notation, and Definitions}

We work in four dimensions. There are three parameters in our model, $g_{0}$, the coupling (at level 0), $N$, (the block spin transformation is for blocks of size $N^{4}$ ), $a$, the measure of plaquette variable smallness. Our lattice is bounded, one may take a single cube as the lattice at level 0 . (See the discussion at the end of this section.) We work with a Lie group, $G$.

The levels or scale sizes are labelled by a parameter $r, r=0,1,2, \ldots$ The edge size at scale $r$ is $1 / N^{r}$. If $r_{2}>r_{1}$ we say level $r_{2}$ is lower scale (level), or finer scale (level), or smaller scale (level) than $r_{1}$. For each scale $r$ there is a lattice, $\mathscr{L}^{r}$. We associate to $\mathscr{L}^{r}$ the coupling constant $g_{r}$,

$$
1 / g_{r}^{2}=1 / g_{0}^{2}+A r+B \ln (r+1) .
$$


The set of plaquettes of $\mathscr{L}^{r}$ we denote as $\mathscr{P}^{r}$, the set of edges of $\mathscr{L}^{r}$ as $\mathscr{E}^{r}$, the vertices of $\mathscr{L}^{r}$ as $\mathscr{V}^{r}$. The vertices of $\mathscr{L}^{r}$ are grouped into blocks of size $N^{4}$, the set of blocks in $\mathscr{L}^{r}$ is called $\mathscr{B}^{r}$. Each block $b$ in $\mathscr{B}^{r}$ has a basepoint $v$ in $\mathscr{V}^{r}$. We write $v=b^{*}$ and $b=v^{*}$. If any vertex $v^{\prime}$ is in $b$ we write, $v^{\prime} \in b$. If $b$ is in $\mathscr{B}^{r}$, then $b^{*}$ is identified with a vertex in $\mathscr{V}^{r-1}$. This gives a $l-1$ identification between elements of $\mathscr{B}^{r}$ and $\mathscr{V}^{r-1}$. We will choose $N$ odd, and pick the base point of each block to be its central vertex.

We are given a universal tree, $T_{\mathrm{UN}}$, on $Z^{4}$ that is maximal (passing through every vertex) and "radial" as defined in [4]. We may later make further requirements on our universal tree. If $v$ is a vertex in $\mathscr{F}^{*}$, we let $t_{v}$, be the radial tree at $v$, a translate and scaling of $T_{\mathrm{UN}}$, that brings the origin in $Z^{4}$ to $v$ and scales by a factor $1 / N^{r}$. If $b$ is in $\mathscr{B}^{r}$, we let $t_{b}$ be the radial tree at $b^{*}$, restricted to $b$. The block transformations in the small field region (the Balaban average, the average before modification as in [4] in the presence of large fields) are defined using the set of $t_{b}$ : If $v_{1}$ and $v_{2}$ are in $\mathscr{V}^{r}$ we write $t_{v_{1} v_{2}}$ for the portion of $t_{v_{1}}$ connecting $v_{1}$ and $v_{2}$ (this is a unique path from $v_{1}$ to $v_{2}$ ). Generalizing $t_{v_{1} v_{2}}$ we now define the path $t\left(v_{1}, v_{2}, v_{3}, \ldots, v_{n}\right)$, where $v_{1}$ is in $\mathscr{V}^{r}$ and $v_{n}$ is in $\mathscr{V}^{r+n-2} \cdot v_{i}, 2 \leqq i \leqq n-1$, will be basepoints (i.e. $v_{i}=b_{i}^{*}$ for some $b_{i}$ in $\left.\mathscr{B}^{r+i-1}\right)$ and are thus elements of both $\mathscr{V}^{r+i-1}$ and $\mathscr{F}^{r+i-2}$. Then $t\left(v_{1}, \ldots, v_{n}\right)$ will be the path that is made up of $t_{v_{1} v_{2}}, t_{v_{2} v_{3}}, \ldots, t_{v_{n-1} v_{n}}$. (In $t_{v_{i}, v_{i+1}}$ one views $v_{i}$ and $v_{i+1}$ as in $\mathscr{V}^{r+i-1}$.)

A field configuration, or a Yang-Mills field, $\mathscr{F}$, is an assignment to each oriented edge of each lattice of an element of $G$, say $g(e)$ to edge $e$. If $e^{\prime}$ is $e$ with the opposite orientation we must have $g\left(e^{\prime}\right)=g^{-1}(e)$, we also write $e^{\prime}=-e$. The crucial additional compatibility property that $\mathscr{F}$ must satisfy is that for $e \in \mathscr{E}^{r}, g(e)$ must be the appropriate block spin transformation (as defined in [4]) of the assignments $g\left(e_{i}\right), e_{i} \in \mathscr{E}^{r+1}$. Block spin transformations, for us, are specifications of $g\left(e_{\alpha}\right)$ for $e_{\alpha} \in \mathscr{E}^{r}$ in terms of $g\left(e_{\beta}\right)$ for $e_{\beta} \in \mathscr{E}^{r+1}$. We naturally denote $\mathscr{F}^{r}$ as the field configuration at scale $r$, the assignments $g\left(e_{\alpha}\right)$ for $e_{\alpha} \in \mathscr{E}^{r}$. The block spin transformation may then be viewed as a mapping

$$
\mathrm{BS}_{r}: \mathscr{F}^{r} \rightarrow \mathscr{F}^{r-1} \text {. }
$$

Mathematically the field $\mathscr{F}$ is a point in the inverse limit of the system $\left\{\mathscr{F}^{r}, \mathrm{BS}_{r}\right\}$, and thus may be written also as $\mathscr{F}^{\infty} . \mathscr{F}^{\infty}$ may be viewed as the field configuration on the imaginary finest lattice $\mathscr{L}^{\infty}$. One may choose to work with a finest lattice, $\mathscr{L}^{\text {MIN }}$, but we find this alternative less attractive.

In this paper we will not need the form of the functional measure $\int d \mathscr{F}$, but for future purposes and present motivation we include a brief discussion. Basically $\int d \mathscr{F}$ is $\prod_{e} \int d \mu_{e}(g(e))$, where $e$ runs over the set of oriented edges in $\mathscr{E}^{\infty}\left(\mathscr{E}^{\mathrm{MIN}}\right.$ for the faint-hearted) and each $d \mu_{e}$ is normalized Haar measure on $G$. (We let each edge occur in the product with only one orientation.) This formal expression is converted to a more meaningful form by writing

$$
\int d \mathscr{F}=\prod_{e} \int d \mu_{e}(g(e)) \prod_{e} \delta(g(e)-\operatorname{Av}(e)),
$$

where the products over $e$ are as above but over edges in all $\mathscr{E}^{r}, 0 \leqq r<\infty$. The $\delta$ functions force $g(e)$ to be the value $\operatorname{Av}(e)$ assigned to it by the block spin transformation. [If $e$ is in $\mathscr{E}^{r}$, then $\operatorname{Av}(e)$ depends on the $g\left(e_{\alpha}\right), e_{\alpha} \in \mathscr{E}^{r+1}$.] One 
commonly uses the $\delta$ functions to eliminate the integrals over certain bond variables $(g(e))$, giving rise to pesty Jacobians. We note that if the $\delta$ function $\delta(g(e)-\operatorname{Av}(e))$ is used to eliminate a variable, and $e \in \mathscr{E}^{r}$, then the Jacobian depends only on $\mathscr{F F}^{r+1}$, (on bond variables down to the $r+1$ level). So eliminating $\delta$ functions in this way, the right side of (1.3) is a product of integrals, each depending on the variables down to a finite level. $\int d \mathscr{F}_{c}$, in (0.4), is this $\int d \mathscr{F}$, multiplied by a product of $\chi_{S}{ }^{\prime}$ s and $\chi_{L}{ }^{\prime}$ s, one $\chi_{S}$ for each $S$-plaquette and one $\chi_{L}$ for each $L$-plaquette in $c$.

We now introduce some nomenclature to describe some simple geometric configurations in the lattices.

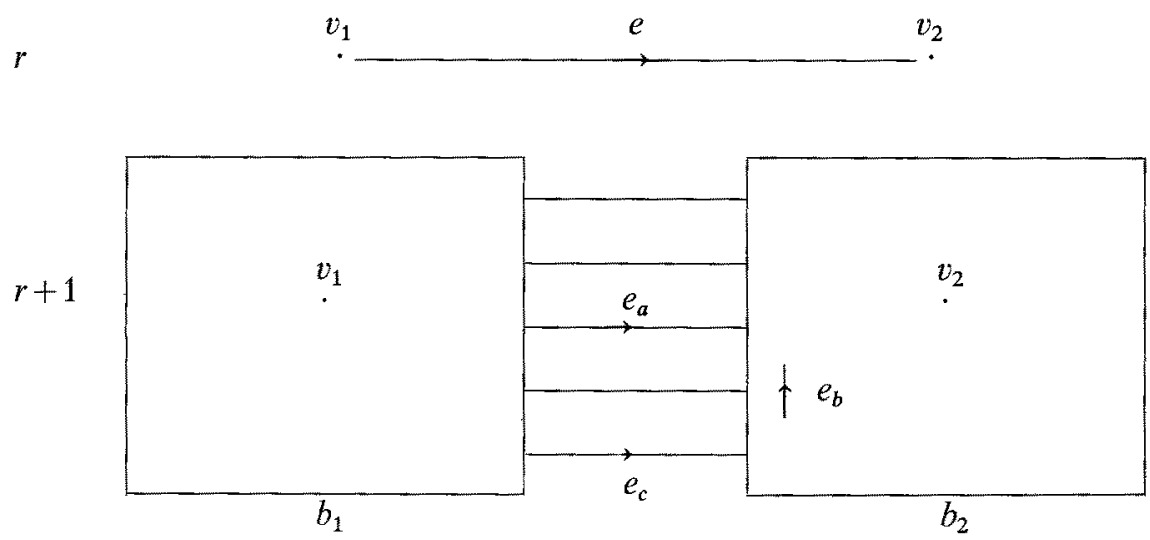

In the figure we see a bond (edge) $e$ in $\mathscr{E}^{r}$ joining $v_{1}$ and $v_{2}$ in $\mathscr{V}^{r} \cdot v_{1}$ and $v_{2}$ are also in $\underset{\mathscr{V}^{r+1}}{\longrightarrow}$ as basepoints, $v_{1}=b_{1}^{*}, v_{2}=b_{2}^{*}$, of blocks $b_{1}$ and $b_{2}$ in $\mathscr{B}^{r+1}$. We write $e=\overrightarrow{b_{1} b_{2}}$ or $e=\overrightarrow{v_{1} v_{2}}$. There are $N^{3}$ edges that join $b_{1}$ and $b_{2}\left(e_{a}\right.$ and $e_{\mathrm{c}}$ are two of them in the figure). We call these channel edges, and their union $\mathscr{E}^{r+1}$ is written $\mathscr{E}_{C} \mathrm{C}$. Edges inside blocks (such as $e_{b}$ in the figure) are called block edges, their union $\mathscr{E}_{B}^{r+1}$. Note $\mathscr{E}_{B}^{r} \cup \mathscr{E}_{C}^{r}=\mathscr{E}^{r}, \mathscr{E}_{B}^{r} \cap \mathscr{E}_{C}^{r}=\emptyset$. In each channel we select a distinguished channel edge, and call it an averaging edge (say $e_{a}$ in the figure), the union of these $\mathscr{E}_{A}^{r+1}$. We call the edges in each block $b$ that lie in $t_{b}$ the identity edges, their union $\mathscr{E}_{I}^{r+1}$. We set $\mathscr{E}_{\mathrm{BM}}^{r}=\mathscr{E}_{B}^{r}-\mathscr{E}_{I}^{r}$, and $\mathscr{E}_{\mathrm{CM}}^{r}=\mathscr{E}_{C}^{r}-\mathscr{E}_{A}^{r}$. The edges in $\mathscr{E}_{\mathrm{BM}}^{r}$ are block mode edges, and those in $\mathscr{E}_{\mathrm{CM}}$ are channel mode edges. We note the disjoint union

$$
\mathscr{E}=\mathscr{E}_{A} \cup \mathscr{E}_{\mathrm{BM}} \cup \mathscr{E}_{\mathrm{CM}} \cup \mathscr{E}_{I}
$$

The notation above is motivated as follows. The integral over the group elements associated to the $e_{a}\left(g\left(e_{a}\right)\right)$ for $e_{a} \in \mathscr{E}_{A}$ will be eliminated by the use of the corresponding "averaging" delta functions in (1.3). In the figure above the integral over $g\left(e_{a}\right)$ would be eliminated by the use of the $\delta$-function $\delta(g(e)-\operatorname{Av}(e))$. The integrals over the group elements associated to edges in $\mathscr{E}_{I}$ will be eliminated, using gauge invariance (these group elements would be set equal to the identity in $G$, in the Balaban axial gauge). There will be a pure mode associated to each edge in $\mathscr{E}_{\mathrm{BM}} \cup \mathscr{E}_{\mathrm{CM}}=\mathscr{E}_{P}$ (except for those that are absorbed into some chunk).

Each mode has associated to it, one edge and a vertex (in some $\mathscr{V}^{r}$ ), its home edge and pinning vertex. Its pinning vertex is the "tail end" of its home edge. The level of a 
mode is the level of its home edge. Each chunk has associated to it a pinning vertex, the effective level of a chunk is the level of its pinning vertex. The level of a chunk is the highest level on which it makes non-trivial assignments. The two levels are set equal for modes. The home edge of a mode is the edge we have previously said is associated to a mode in a 1-1 manner. Pure modes have home edges in $\mathscr{E}_{P}$, and split into channel and block modes by the decomposition of $\mathscr{E}_{P} . A$-modes have home edges in $\mathscr{E}_{A}$, a $1-1$ correspondence. The amplitude of a mode is the group element (or Lie Algebra element) associated to its home edge by its isolated field.

For each given $S-L$ configuration there will be later specified a collection of disjoint subsets of the edges. Each such subset is the set of edges on which a chunk lives. We will say such edges "belong to the chunk" or are "in the chunk". In constructing the field only pure modes whose home edges do not belong to any chunk are kept.

For the action on the $r$ th lattice, $\mathscr{L}^{r}$, we take $\frac{1}{g_{r}^{2}} S_{0}^{r}$, where

$$
S_{0}^{r}=\frac{1}{4} \sum_{p \in \mathscr{P} r} f\left(A_{\partial p}\right)
$$

See (3.2), (3.3), and (3.4) of [4]. The total action is given as

$$
S=\frac{1}{g_{0}^{2}} S_{0}^{0}+\sum_{r=1}\left(\frac{1}{g_{r}^{2}} S_{0}^{r}-\frac{1}{g_{r-1}^{2}} S_{0}^{r-1}\right)=S_{R}+S_{0},
$$

where

$$
\begin{gathered}
S_{R}=\sum_{r=1}\left(\frac{1}{g_{r}^{2}}-\frac{1}{g_{r-1}^{2}}\right) S_{0}^{r-1}, \\
S_{0}=\frac{1}{g_{0}^{2}} S_{0}^{0}+\sum_{1} \frac{1}{g_{r}^{2}}\left(S_{0}^{r}-S_{0}^{r-1}\right),
\end{gathered}
$$

$S_{R}$ is viewed as the renormalization counter terms. $S_{0}$ may be viewed as the unrenormalized action. Loosely speaking we will later take the "quadratic part" of $S_{0}$ to be a "free action".

Finite Volume Effects, A Technical Digression. Since we do not know how to handle infra-red problems in gauge theories, we must work with a finite size system. One possibility is to use periodic boundary conditions, a periodic lattice. We prefer not to follow this course. It is rather artificial, and introduces geometric differences from the infinite volume situation. If we merely use a bounded lattice (free boundary conditions), then the propagators will feel long range effects near the boundary of the region. (We have not considered other possibilities, such as Dirichlet boundary conditions, but these would also not meet the next objection.) We would like the (isolated) modes, in the small field region, to have their infinite volume form, as in $[2,3]$.

We would prefer to follow a route that is much like the imposition of boundary conditions in the work of Brydges and Federbush on Debye screening. There is an infinite volume lattice (at all levels). Outside a fixed volume, $V_{1}$, all plaquettes are $S$ plaquettes. Outside a larger volume, $V_{2}$, all pure mode amplitudes are zero. Outside 
a larger volume $V_{3}$ averaging corrections are not performed. (This last step is more aesthetic to omit. There will then be an infinite number of averaging corrections at each level, but this is harmless.) The action will receive contributions only from plaquettes inside volume $V_{3}$. In fact notationally we write most of the paper in the notation of a finite lattice situation. (We do not address the fact that sometimes our trees leave the finite lattice, an easy to correct problem. We do require $t_{b}$ to lie in $b$. The treatment of the boundary we really have in mind (involving volumes $V_{1}, V_{2}$, and $V_{3}$ as above) is easily translated to a pure finite volume notation, and vice versa. The proofs will follow the $V_{1}, V_{2}, V_{3}$ treatment of the boundary.

\section{The Isolated Pure Mode}

In this section we define the isolated field due to a single pure mode, other degrees of freedom (modes or chunks) are assumed unexcited. Thus we are in a $S-L$ configuration with only $S$-plaquettes at all levels.

We first consider a channel mode of level $r$. We call the mode $m$, and the associated edge in $\mathscr{E}_{\mathrm{CM}}$ by $e(m) . e(m)$ is the home edge of $m$. In the figure, showing

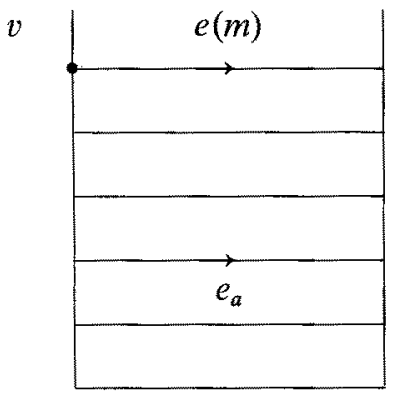

the channel containing $e(m)$, let $e_{a}$ be the averaging edge, $e_{a}$ is the home averaging edge of $m$, and we write it as $\bar{e}(m), v$ will be the pinning (vertex) of $m$. We write this as $v(m)$. We pick like preferred orientations for all edges parallel to a given direction over the whole of all the lattices. (Say the plus $x$-direction for edges parallel to an $x$-axis.)

We now start to define the field configuration of the mode $m$. We write $g(m, e)$ for the group element assigned to the edge $e$ by the isolated mode $m$. We also write

$$
g(m, e)=e^{A(m, e)}
$$

$A(m, e)$, an element of the Lie algebra of $G$, will be small. If $e$ is in $\mathscr{E} r^{\prime}$ with $r^{\prime}<r$, then $A(m, e)=0$. If $e$ is in $\mathscr{E}^{r}$, but $e \neq e(m), e \neq \bar{e}(m)$, then $A(m, e)=0$. On the home and home averaging edges we have

or equivalently

$$
g(m, e(m))=g^{-1}(m, \bar{e}(m))
$$

$$
A(m, e(m))=-A(m, \bar{e}(m))
$$

(for orientations as in the figure!) The value of $g(m, e(m)$ ) [or equivalently of 
$A(m, e(m))]$ will be integrated over in the functional integral. $g(m, e(m))$ and $A(m, e(m))$ will both be called the amplitude of the mode $m$. We temporarily defer describing the field for levels below $r$. We make the observation that the assignments we have so far made are consistent with the averaging procedure. The averaging in a situation where all group elements commute and are small is exactly the usual averaging of Lie algebra elements (associated to the paths involved in the averaging procedure).

We now turn to a block mode $m$ at level $r$. In the figure, as in the last case, $e(m)$ in $\mathscr{E}_{\mathrm{BM}}$ is the home edge of $m, v$ the pinning vertex $v=v(m)$. If $e^{\prime}$ and $e^{\prime \prime}$ in the figure are both in $\mathscr{E}_{A}$, and parallel to $e(m)$ as indicated, then $e^{\prime}$ and $e^{\prime \prime}$ are the two home

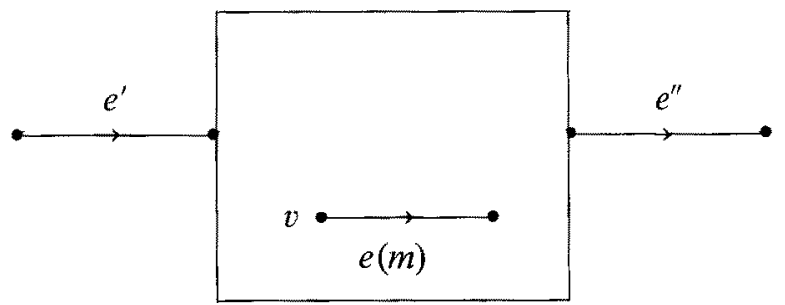

averaging edges of $m,\left(\bar{e}_{1}\right.$ and $\left.\bar{e}_{2}\right)$. We now turn to defining the field configuration for this mode. For $e$ in $\mathscr{E}^{r^{\prime}}$, with $r^{\prime}<r, A(m, e)=0$. If $e$ is in $\mathscr{E}^{r}$, but $e \neq e(m), e \neq \bar{e}_{1}(m)$, $e \neq \bar{e}_{2}(m)$, then $A(m, e)=0$. On the home and home averaging edges we have

$$
\begin{aligned}
& A\left(m, \bar{e}_{1}(m)\right)=-k_{1} A(m, e(m)), \\
& A\left(m, \bar{e}_{2}(m)\right)=-k_{2} A(m, e(m)),
\end{aligned}
$$

where $k_{1}$ and $k_{2}$ are numerical factors depending only on the position of $e(m)$ in the block. $k_{1}$ and $k_{2}$ are chosen so that the assignments so far are consistent with the averaging procedure (we are in a small field region). $A(m, e(m))$ and $g(m, e(m))$ are both the amplitudes:

We now have the $A$ field assignments at the $r$ level for both kinds of pure modes, zero except for the values in (2.3) and (2.4). [2,3] present a continuum $A$ field of this mode consistent with these assignments, as well as assignments to each edge. (The continuum field does not minimize the "quadratic" terms in $S_{0}$, the "free action", but rather this "free action" in the "approximation" that all the $g_{r}$ 's are equal. This is technically easier:)

Note. For our modes in this section all the $A$ 's are proportional to the same Lie algebra element and thus we may treat them as (commuting) numerical quantities. The calculations for these quantities is the same as in $[2,3]$.

\section{The Isolated $\boldsymbol{A}$-Mode}

Let $m$ be an $A$-mode of level $r$, so its home edge $e(m)$ is in $\mathscr{E}_{A}^{r}$. Its amplitude is $A(m, e(m))$ [or $g(m, e(m))]$. We obtain from $[2,3]$ a continuum field of the mode consistent with assignments on level $r$ of $A(m, e(m))$ to $e(m)$ and of zero to other 
edges in $\mathscr{E}^{r}$. [2,3] also yields assignments to all edges. But, for all edges $e$ in $\mathscr{E}^{r^{\prime}}, r^{\prime}<r$, we set $A(m, e)=0$. This yields assignments that violate averaging at a single edge. As with $p$-modes all assignments and the continuum field are multiples of a single Lie Algebra element.

\section{Ordering of Excitations}

Since our Yang-Mills field will be constructed by an inductive process of laying down excitations one at a time, it is necessary to decide on the order in which this takes place. We select an ordering on excitations, the Universal Excitation Ordering. We will write $E_{1}<E_{2}$ if excitation $E_{1}$ occurs before excitation $E_{2}$ in this ordering. The ordering will have the following properties:

O1) If excitation $E_{1}$ is lower effective level than excitation $E_{2}$ then $E_{2}<E_{1}$. (Recall lower level corresponds to higher $r$ value, shorter length scale.)

O2) If $E_{1}$ and $E_{2}$ have the same effective level, and $E_{1}$ is a $p$-mode and $E_{2}$ is not, then $E_{1}<E_{2}$.

O3) If $E_{1}$ and $E_{2}$ have the same effective level, and $E_{1}$ is an $A$-mode and $E_{2}$ is not, then $E_{2}<E_{1}$. (The $A$-modes are last laid down at a given level, ensuring averaging holds; this determines each $A$-mode's amplitude at the time it is added to the field;)

O4) The ordering of chunks, of the same effective level, is determined depending on their pinnings, and edges belonging to them. The ordering of modes is by their home edges. (Ordering of excitations is determined by their "geometric" content:)

We have ordered the set of all possible excitations (we have a total ordering). In any given $S-L$ configuration only some of the chunks appear, and some of the $p$ modes may be absent. This subset will be ordered (by the subset ordering) and is the order in which this subset of excitations is laid down in the field construction.

\section{Prelude to Section 6}

We are given a fixed $S-L$ configuration: We will later specify for this configuration its collection of associated (disjoint) chunks. The field will be constructed from these chunks, all $A$-modes, and those $p$-modes whose home edges are not in any of these chunks. These are ordered, as a subset of all excitations, by the Universal Excitation Ordering. We denote this ordered sequence as

$$
E_{1}, E_{2}, \ldots \text {. }
$$

We have already discussed the isolated field of mode excitations. The isolated fields of chunks will be later specified. We denote the isolated fields of the excitations in (5:1) by

$$
\mathscr{C}_{1}, \mathscr{C}_{2}, \ldots
$$


For excitation $E_{i}$ we introduce notation for some of its associated quantities:

Isolated field assignment to edge $e$

$$
A\left(E_{i}, e\right) \text { or } g\left(E_{i}, e\right),
$$

Isolated Continuum field

$$
A_{\mu}\left(E_{i}, x\right),
$$

Home edge (of a mode)

Home Averaging edge

$$
e\left(E_{i}\right)
$$

$$
\begin{array}{cl}
\bar{e}\left(E_{i}\right) & \text { for channel mode } \\
\bar{e}_{1}\left(E_{i}\right), \bar{e}_{2}\left(E_{i}\right) & \text { for block mode }
\end{array}
$$

Pinning vertex

$$
v\left(E_{i}\right) \text {. }
$$

We may later introduce obvious shorthands, such as

$$
A(i, e) \text { for } A\left(E_{i}, e\right) \text {. }
$$

For each level, $r$, we will need a partition of unity with functions labelled by vertices in $\mathscr{V}^{r}$. We first discuss a partition of unity on $R^{4}$ with functions labelled by points $\alpha \in Z^{4}$.

$$
1=\sum_{\alpha \in Z} \phi_{\alpha}(x), \quad x \in R^{4} .
$$

The $\phi_{\alpha}$ are $C^{\infty}$ with the properties

1) $\left\{\phi_{\alpha}(x)\right\}$ is translation invariant. That is, $\phi_{\alpha}(x)=\phi(x-\alpha)$ for some function $\phi(x)$.

2) $\phi(x)$ is invariant under the discrete symmetries of the lattice $Z^{4}$ (with origin fixed). (This requirement is not necessary.)

3) $\phi(0)=1$,

4) $\phi(x)=0$ if $|x|>2$.

In $\mathscr{L}^{0}$ each vertex (in $\mathscr{V}^{0}$ ) corresponds to one of the $\phi_{\alpha}$. By scaling the partition of unity, we get a partition of unity, for each $r$, with elements associated to the vertices in $\mathscr{V}^{r}$. [One is replacing $\phi(x)$ by $\phi\left(N^{r} x\right)$.] If $v \in \mathscr{V}^{r}$ we write $\phi_{v}(x)$ as the appropriate function in the scale $r$ partition of unity.

\section{Gauge Invariant Coupling - Laying Down an Excitation}

This section introduces the most important and the cleverest idea in this paper. It is the incorporation of the field of an isolated excitation into the total field. Recall that the total field is developed inductively, introducing the effects of pure modes, chunks, and averaging correction modes, in sequence, one at a time. In this section we assume that the total field has been developed to some stage, and then we introduce the additional contribution of a single excitation. The field as it has developed to this point has contributions from excitations at larger (or equal) 
effective scale than the excitation we are about to introduce. (Recall the principle mentioned in the introduction of excitations being developed in a "background field of larger scale excitations:") All our definitions will imply a simple addition of the $A$ fields of the different excitations to linear order in the $A$ 's.

To be specific, we have laid down the first $n-1$ excitations, and are about to describe the incorporation of $E_{n}$ into the field, i.e. we know $\mathscr{F}_{n-1}$,

$$
\mathscr{\mathscr { F }}_{n-1}=\mathscr{C}_{n-1} \times \mathscr{C}_{n-2} \times \ldots \times \mathscr{C}_{1}
$$

and we wish to define

$$
\mathscr{F}_{n}=\mathscr{C}_{n} \times \mathscr{F}_{n-1}
$$

We have previously introduced the (abbreviated) notation of $g(i, e)$ for the assignment of $\mathscr{C}_{i}$ (or $E_{i}$ ) to edge $e$.

$G(n-1, e)$ is the bond variable (assignment) at edge $e$ due to the first $n-1$ excitations, and $g(n, e)$ is the assignment of the isolated field of $E_{n}$ to edge $e$. We are about to come up with a better form of their combined field assignment for $e$ than $g(n, e) G(n-1, e)-$ one incorporating the idea of gauge invariant coupling. The assignment we will get will be $G(n, e)$. We let $r$ be the effective level of $E_{n}$, and separately treat the three cases, with $e \in \mathscr{E}^{r^{\prime}}$, of $r^{\prime}<r, r^{\prime}=r, r^{\prime}>r$.

$r^{\prime}<r$. In this case we simply set

$$
G(n, e)=G(n-1, e) .
$$

$r^{\prime}=r$ : This case exposes the essential features of the gauge invariant coupling procedure, without the technical difficulties of the next case. Were we simply to set $G(n, e)=g(n, e) G(n-1, e)$ say, then even if $\mathscr{F}_{n-1}$ were a pure gauge field (that is, even if $G(n-1, \partial p)=$ Id for all $p$ in $\mathscr{P}^{r}$, in a clear notation for a plaquette assignment) one would not necessarily have $|G(n, \partial p)|=|g(n, \partial p)|$ for all $p$ in $\mathscr{P}^{r}$. This we desire. We are still going to set

$$
G(n, e)=G(n-1, e)
$$

for all $e$ for which $g(n, e)=\mathrm{Id}$. (This will always be true.) We will use $t_{v(n)}$ [as defined before, the scaling and translation of the universal tree, $T_{\mathrm{UN}}$, to scale $r$ and "origin" $v(n)]$. Let $e$ be an edge in $\mathscr{E}^{r}$ whose tail end is $v(n)$ (one of eight such). We then set

$$
G(n, e)=g(n, e) G(n-1, e)
$$

and illustrate this with a trivial figure.

$$
\underset{v(n) \quad \vec{e}}{\stackrel{g(n, e) \cdot G(n-1, e)}{e}} .
$$

Note that the orientation of the edge and the order of $g$ 's in the product will be crucial, Now let $e \in \mathscr{E}^{n}$ be an arbitrary edge. We let $e=\overrightarrow{v_{a} v_{b}}$ and consider the portion of $t_{v(n)}$ between $v(n)$ and $v_{a}, t_{v(n) v_{a}}$. This is illustrated in the figure with 


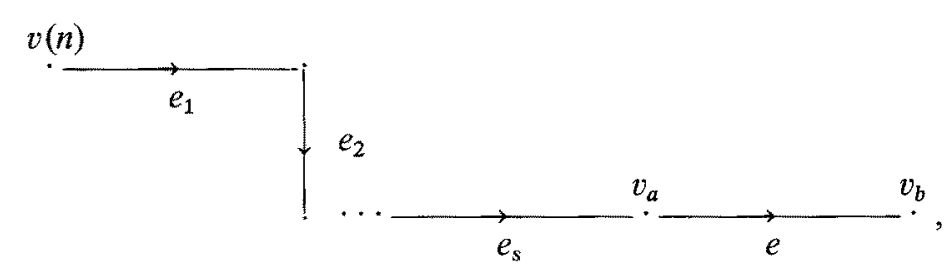

$e_{1}, e_{2} \ldots e_{s}$ the path $t_{v(n) v_{a}}$; note the orientations we are using. Using the figure we now define

$$
u(e)=G\left(n-1, e_{1}\right) G\left(n-1, e_{2}\right) \ldots G\left(n-1, e_{s}\right)
$$

and in terms of $u(e)$, set

$$
G(n, e)=u^{-1}(e) g(n, e) u(e) G(n-1, e) .
$$

We leave to the reader to check that with this definition we have achieved our purpose of making pure gauge background fields not contribute to plaquette variables. The coupling is to the " $F$ " field not the " $A$ " field of the background. The excitation $E_{n}$ is "grounded" at $v(n)$, its pinning, "pure gauges gauged away from this point along $t_{v(n)}$.

$r^{\prime}>r$. One is tempted from the $r=r^{\prime}$ situation to again make use of a tree centered at the pinning vertex of the excitation and to make exactly analogous definitions of the $G(n, e)$ field on the lower scale edges. This agrees with the gauge invariant coupling principle, but it has other technical difficulties. In the case that $G(n-1, e)$ is not a pure gauge field, the $G(n, e)$ field so generated would not be sufficiently regular as the scales get finer and finer. The paths through the tree for points arbitrarily close may be rather different, leading to too large plaquette variables. A solution to this technical difficulty, that has all the ingredients of the $r=r^{\prime}$ case, is to "average" the $G(n, e)$ over different choices of trees in a suitable way. Such averaging is just like the sort of slightly unpleasant technical device one must use all through this cluster expansion - the block spin transformation averaging. In both cases one would rather not average if one could avoid it. On the other hand one can get to like these solutions of technical problems.

We use now the notation from Sect: $1 t\left(v_{1}, v_{2}, \ldots, v_{s}\right)$ for a path as therein described. We use $\Gamma$ to label this path, a sequence of edges of different sizes, living in different lattices. But just as the sequence of edges $e_{1}, e_{2}, \ldots, e_{s}$ in the last figure gave rise to a product of group elements, a $u(e)$, so $\Gamma$ gives rise to a product of group elements, and this product we call $u(\Gamma)$. We associate to $\Gamma=t\left(v_{1}, v_{2}, \ldots, v_{s}\right)$ the weighting

$$
w(\Gamma)=\phi_{v_{2}}(\hat{e}) \phi_{v_{3}}(\hat{e}) \ldots \phi_{v_{s-1}}(\hat{e})
$$

where we here view $v_{2} \in \mathscr{V}^{r}, v_{3} \in \mathscr{V}^{r+1}, \ldots, v_{s-1} \in \mathscr{V}^{r+s-3}$ in deciding the scales of the $\phi$ 's. $\hat{e}$ is the center of $e$. Note that

$$
\sum_{\Gamma} w(\Gamma)=1
$$


where we fix $v_{1}$ and $v_{s}$ in the sum. We now generalize the averaging procedure of Sect. 1 [4]. Refer to this section now.

At this point we follow a slightly different procedure for modes and for chunks.

Procedure for Modes. We define $G_{I}(n, e)$ by

$$
u^{-1}(\Gamma) g(n, e) u(\Gamma) G(n-1, e),
$$

and find $G(n, e)$ by minimizing

$$
\sum_{\Gamma} w(\Gamma) d^{2}\left(G(n, e), G_{\Gamma}(n, e)\right)
$$

Procedure for Chunks. We define $\bar{u}$ by minimizing

We then set

$$
\sum_{\Gamma} w(\Gamma) d^{2}(\bar{u}, u(\Gamma))
$$

$$
G(n, e)=\bar{u}^{-1} g(n, e) \bar{u} G(n-1, e) .
$$

$\bar{u}$ depends on $n$ and $e$, and may be denoted as $\bar{u}(n, e)$.

In both cases we will operate in a situation where the indicated minima are unique.

Note. One carries out the construction of laying down group assignments to an edge $e$ with $e$ in its preferred orientation, ( $e$ in the two figures of this section). Afterwards one sets $g(-e)=g^{-1}(e)$.

\section{Gauge Invariant Coupling - An Epiphany}

We write $\mathscr{F}^{\mathscr{G}}$ for a gauge transformation $\mathscr{G}$ of $\mathscr{F}$. We desire the result of a gauge transformation on an inductively constructed field. We let $h_{v}(\mathscr{G})$ be the group element assigned to vertex $v$ by $\mathscr{G}$. (A gauge transformation is exactly an assignment for all vertices of an element of $G$. When $v$ may be identified as a vertex in several $\mathscr{V}^{r}$, the assignment to it in all its occurrences must be identical. This is a compatibility under gauge transformations that we have enforced by the gauge invariance of our block spin transformations.) If $\mathscr{C}_{i}$ is the isolated field of an excitation, we will write $\mathscr{C}_{i}^{g}$ for the isolated field that is obtained by transforming each edge assignment $g(i, e)$ to the assignment $g g(i, e) g^{-1}$ (for a fixed $g \in G$ ). We abbreviate $\mathscr{C}_{i}^{h_{v(i)}\left(\mathscr{t}^{2}\right)}$ by $\mathscr{C}_{i}^{t(\mathfrak{t})}$. We obtain the basic result

Gauge Twist Relation 1.

$$
(\mathscr{C} \times \mathscr{F})^{\mathscr{G}}=C^{t(\mathscr{G})} \times \mathscr{F}^{\mathscr{G}},
$$

from which may be obtained by induction

Gauge Twist Relation 2.

$$
\left(\mathscr{C}_{n} \times \ldots \times \mathscr{C}_{1}\right)^{\mathscr{G}}=\mathscr{C}_{n}^{t(\mathscr{G})} \times \ldots \times \mathscr{C}_{1}^{t(\mathscr{G})} \times 1^{\mathscr{G}} .
$$

1 is the "trivial" field all of whose edge assignments are the identity. 
We note $\mathscr{C}_{i}^{g}$ may be obtained from $C_{i}$, for a mode, by merely changing its amplitude $g(i, e(i))$ to $g g(i, e(i)) g^{-1}$. We will construct chunk fields defining their internal variables so that $\mathscr{C}_{i}^{g}$ may be obtained by merely changing each internal variable from $g_{\alpha}$ to $g g_{\alpha} g^{-1}$.

One cannot deny an inner harmony evident in the Gauge Twist Relations (7.1) and (7.2).

\section{The Geometric Content of the Chunks}

We assume a fixed $S-L$ configuration, and in this section specify the geometric content of the associated set of chunks. Preliminary to specifying chunks, we develop intermediate objects, hunks.

Let $p$ be an $L$-plaquette in some $p^{r}$. We let $\mathscr{M}(p)$ be the set of edges, in $\mathscr{E}^{s}, r-N_{c r}$ $\leqq s \leqq r+1, N_{c r}$ to be specified later, determined as the minimal set satisfying:

1) At each level $\left(r-N_{c r} \leqq s \leqq r+1\right)$ it is a union of blocks and channels.

2) At each such level it contains all edges within distance $\left(N_{c r}+C_{1 c}\right) N^{-s}$ of $p$. $\left(C_{1 c}\right.$ to be later specified.)

We say $\mathscr{M}\left(p_{1}\right)$ and $\mathscr{M}\left(p_{2}\right)$ "overlap" if they share an edge. This provides an idea of connectivity on the $\{\mathscr{M}(p)\}$ (where $p$ runs over all $L$-plaquettes). The hunks are the connected components of this set. Thus each hunk is some union of elements $\mathscr{A}\left(p_{i}\right)$, the $p_{i} L$-plaquettes.

Each chunk will be a union of hunks, the chunks will be disjoint, and their union will be the union of hunks. Thus specifying the chunks is equivalent to determining a partition of the set of hunks. This partition is selected as a finest partition (not necessarily unique?) for which the following property holds.

Well-Separation of Chunks. Let $E$ be a chunk of diameter $d(E)$. We let $\mathscr{E}_{u}(E)$, the umbrella edges of $E$, be the union of edges of length $\leqq C_{2 c} d(E)$, and at distance $\leqq C_{2 c} d(E)$ from $E$. $\left(C_{2 c}\right.$ will be later specified.) Then if $E^{\prime}$ is any chunk with $d\left(E^{\prime}\right) \geqq d(E)$, the edges of $E^{\prime}$ do not intersect the edges in $\mathscr{E}_{u}(E)$.

This property (for $C_{2 c} \gtrsim 1$ ) will guarantee that in the process of laying down the isolated field of $E$, the paths $\Gamma$ appearing in (6.9) with positive weight do not pass through the edges of any other chunk whose field is already laid down.

Note that in distance, as above (determining the diameter of $E$, the distance between $E$ and an edge) all objects are viewed living in $R^{4}$. We will always mean that by distance. Thus $d\left(e_{1}, e_{2}\right)$ is well defined even if $r\left(e_{1}\right) \neq r\left(e_{2}\right)$.

The level $r(E)$ of a chunk $E$ is the level of its highest level edges. The effective level $r_{e}(E)$ of a chunk is the largest $r^{\prime}$ such that

$$
N^{-r^{\prime}} \geqq d(E)
$$

with $d(E)$ the diameter of the chunk. [We do not let $r_{e}(E)$ be negative, if $(8: 1)$ selects a negative number we set $r_{e}=0$.] $v(E)$, the pinning vertex, is in $\mathscr{V}^{r_{e}}$, and chosen at distance $<N^{-r_{e}}$ from $E$.

\section{The Isolated Chunk Field}

In this section we present the construction of the isolated chunk field of a chunk $E$. In fact, our construction involves two subroutines that are detailed in later sections. 
Using these, the field is developed by an inductive process. (Recall that the isolated field of a pure mode is developed as determined by a single group element, its amplitude. The assignments of the isolated pure mode were nontrivial to arrive at $[2,3]$; it is not surprising that the field of a chunk, developed as determined by many group elements, its internal variables, is highly non-trivial to construct:) The chunk has level $r(E)$, its isolated field assignment to edge $e$ will be $g(E, e)$, which we will abbreviate by $g(e)$.

The assignments we find will be restricted by the following criterion determined by the $S-L$ configuration in which the chunk appears. For $p$ an $S$-plaquette we will require $\left|g_{\partial p}\right|<c a$, for $p$ an $L$-plaquette inside the chunk we will require $\left|g_{\partial p}\right|>\frac{a}{8}$ (the $g_{\partial p}$, isolated field values).

The two subroutines we use are Field Interpolation (developed in Sect: 10) and Gauge Interpolation (developed in Sect. 11). The $g(e)$ are determined level by level (from higher level down). Assignments are developed by a sequence of approximations. We will find a sequence of assignments to $e, g(e, r), r \leqq r(e)$ such that

$$
g(e)=g(e, r(e)) \text { if } \quad e \in \mathscr{E}_{I} .
$$

We introduce $g^{\delta}(\cdot, \cdot)$ such that

$$
g(e, r)=g^{\delta}(e, r) g(e, r-1) .
$$

Global Gauge Requirement. If the internal variables of a chunk are modified by sending $g \rightarrow h g h^{-1}$, for some fixed $h$, then the isolated field assignment to any edge $e$ likewise is changed $g(e) \rightarrow h g(e) h^{-1}$. (This is automatic for $e \in E-\mathscr{E}_{A}-\mathscr{E}_{I}$ :)

Let $\left\{g_{i}\right\}$ be the (ordered) set of (independent) internal variables, determining our chunk. We view the action of $G$ on this set $\left\{g_{i}\right\}^{h}=\left\{h g_{i} h^{-1}\right\}$. We may determine the isolated chunk field for one point in each orbit of this action, and use the Global Gauge Requirement to determine the isolated chunk field for any set of variables. We implicitly follow this route to unobtrusively ensure the Global Gauge Requirement (we need not mention this in the course of our construction).

We proceed to the detailing of the chunk isolated field construction, specifying $g(e)$ and $g(e, r)$.

$r(e)<r(E)$. Here we set

$$
g(e)=\mathrm{Id} \quad \text { if } \quad r(e)<r(E) .
$$

$r(e)=r(E)$. We now set

$$
\begin{gathered}
g(e)=\mathrm{Id} \text { if } e \in \mathscr{E}_{I}^{r(E)}, \\
g(e)=\mathrm{Id} \text { if } r(e)=r(E), \quad e \notin E, \quad e \notin \mathscr{E}_{A} .
\end{gathered}
$$

We mean $e \in E$ to mean $e$ is in the set of edges of the geometric chunk (as given in Sect. 8). If $e$ is in $E$, of level $r(E)$, and not in $\mathscr{E}_{I}$ or $\mathscr{E}_{A}$ it is assigned an internal variable of the chunk (freely specified subject to plaquette variable restrictions mentioned above). Assignments to edges in $\mathscr{E}_{A}$ are determined to satisfy averaging. We finally set

$$
g(e, r)=\mathrm{Id}, \quad \text { for } \quad r \leqq r(E)
$$


The Induction Step. We assume $g(e)$ known for $r(e) \leqq r$, and $g\left(e, r^{\prime}\right)$ known for $r^{\prime} \leqq r$. We desire to find $g(e)$ for $r(e)=r+1$, and $g(e, r+1)$. As above we need not consider $e \in \mathscr{E}_{A}^{r+1}$ and $e \in\left(E-\mathscr{E}_{I}-\mathscr{E}_{A}\right), r(e)=r+1$.

Gauge Interpolation will determine $g(e, r+1)$ [and thus $g(e), e \in \mathscr{E}_{I}^{r+1}$ ], and dependent on those assignments, Field Interpolation will specify $g(e)$ for

$$
\left\{e, e \notin E, e \notin \mathscr{E}_{A}, e \notin \mathscr{E}_{1}, r(e)=r+1\right\} .
$$

\section{Field Interpolation}

We assume known $g(e)$ for $e \in \mathscr{E}^{r}$, and $e \in\left(\mathscr{E}^{r+1} \cap E-\mathscr{E}_{A}\right)$, and for $e \in \mathscr{E}_{I}^{r+1}$. These latter assignments will be given in Sect. 11. We desire to determine $g(e)$ for all $e \in \mathscr{E}^{r+1}$ [actually we need only determine assignments for $e \in\left(\mathscr{E}^{r+1}-\mathscr{E}_{A}-\mathscr{E}_{I}-E\right)$ ]. We let $\mathscr{V}_{c}^{r}$ be the set of vertices in $\mathscr{V}^{r}$ outside $E$. We will later want a $C^{\infty}$ partition of unity associated to these vertices, $\left\{\phi_{v}^{\prime}(x)\right\}$, such that

We require

$$
\phi_{v}^{\prime}(x)=0 \text { for } d(x, v)>4 N^{-r} .
$$

$$
\sum_{v \in \mathscr{F}_{c}^{\prime}} \phi_{v}^{\prime}(x)=1
$$

outside $E \cap \mathscr{L}^{r}$ (here viewing this as a solid set in $R^{4}$ ).

For each $v \in \mathscr{W}_{c}^{r}$ we will find an assignment $g^{v}\left(e^{\prime}\right)$ to each edge $e^{\prime}$ in $\mathscr{E}^{r+1}$ outside $E$. We now pick an edge assignment $g\left(e^{\prime}\right)$ that is a suitable average of these:

defined by minimizing

$$
g\left(e^{\prime}\right)=\operatorname{Av}_{v} g^{v}\left(e^{\prime}\right)
$$

$$
\sum_{v} \phi_{v}^{\prime}\left(e^{\prime}\right) d^{2}\left(g\left(e^{\prime}\right), g^{v}\left(e^{\prime}\right)\right)
$$

where $\hat{e}^{\prime}$ is the center of edge $e^{\prime}$. The assignments $g\left(e^{\prime}\right)$ determined above may not satisfy averaging. But we use these values for edges $e^{\prime}$ in $\mathscr{E}^{r+1}-\mathscr{E}_{A}^{r+1}$, the assignments to edges in $\mathscr{E}_{A}^{r+1}$ chosen to guarantee consistency with averaging.

We turn to the task of specifying the $g^{v}\left(e^{\prime}\right)$. Let $t_{v}$ be the tree in $\mathscr{L}^{r}$ centered at $v$, as previously defined. We switch to a gauge where the assignments to the bonds of $t_{v}$ are trivial $(=\mathrm{Id})$. For any lattice vertex, $v^{\prime}$, we set $u\left(v^{\prime}\right)=u\left(t_{v v^{\prime}}\right)$, the $u^{\prime}$ s as in Sect. 6 (with a slight change in notation). The assignment in the new gauge, $h(e)$, for $e=\overrightarrow{v_{a} v_{b}}$ in $\mathscr{L}^{r}$ is

We write

$$
h(e)=u\left(v_{a}\right) g(e) u^{-1}\left(v_{b}\right)
$$

$$
h(e)=e^{\sum_{\alpha} A^{\alpha}(e) L_{\alpha}},
$$

where the $\left\{L_{\alpha}\right\}$ are a basis for the Lie Algebra, and the $A^{\alpha}(e)$ are "small". We will only use $h(e)$ for $e$ "close" to $v$. We will explicitly want

$$
d(e, v)<\left(\frac{C_{1 \mathrm{c}}}{2}\right) \cdot N^{-r} .
$$


We fix $\alpha$ and look at the assignments to the edges of $\mathscr{L}^{r}$ (in terms of $A$ 's). We first split $A^{\alpha}$ into a sum

$$
A^{\alpha}(e)=A_{0}^{\alpha}(e)+A_{\delta}^{\alpha}(e) .
$$

Both $A_{0}^{\alpha}$ and $A_{\delta}^{\alpha}$ are in the radial axial gauge about $v$, equal zero on edges in $t_{v}: A_{0}^{\alpha}$ yields plaquette values constant on all parallel plaquettes. In each of the six plaquette orientations there is a plaquette, one of whose vertices is $v$, such that $A^{\alpha}$ and $A_{0}^{\alpha}$ assign the same plaquette values to it. ( $A_{0}^{\alpha}$ is a constant curvature field matching $A^{\alpha}$ at $v$, and $A_{\delta}^{\alpha}$ is a fluctuation from this constant field. The smoother $A^{\alpha}$ is, the smaller is $A_{\delta}^{\alpha}$.) We now set

$$
\begin{aligned}
A(e) & =A^{\alpha}(e) \quad \text { if } \quad d(e, v)<\left(\frac{C_{1 \mathrm{c}}}{2}\right) \cdot N^{-r} \\
& =A_{0}^{\alpha}(e) \quad \text { if } \quad d(e, v) \geqq\left(\frac{C_{\mathbf{c}}}{2}\right) \cdot N^{-r} .
\end{aligned}
$$

We use $[2,3]$ to determine a distribution on the edges of $\mathscr{L}^{r+1}$, determined as minimizing the continuum actions subject to the constraint of satisfying (10.7). We will use only the corresponding plaquette assignments on $\mathscr{L}^{r+1}$, deriving edge assignments as follows.

Using the tree $t_{v}$, where $\dot{v}$ is $v$ as viewed in $\mathscr{V}^{r+1}$, we can find unique $A^{\alpha}\left(e^{\prime}\right)$, $e^{\prime} \in \mathscr{E}^{r+1}$, such that $A^{\alpha}\left(e^{\prime}\right)=0$ if $e^{\prime}$ is in $t_{\hat{\theta}}$, and the plaquette assignments are as above: We define

$$
\tilde{h}_{v}\left(e^{\prime}\right)=e^{\sum_{\alpha} A^{\alpha}\left(e^{\prime}\right) L_{\alpha}} .
$$

(Note that while we work with $\alpha$ fixed above, one is working in an abelian situation (artificially).)

We now define $\left\{g^{v}\left(e^{\prime}\right)\right\} \cdot g^{v}\left(e^{\prime}\right)$ is a gauge transformation of $\tilde{h}_{v}\left(e^{\prime}\right)$. There are $\psi\left(v^{\prime}\right) \in G$, for $v^{\prime}$ in $\mathscr{q}^{r+1}$, such that if $e^{\prime}=\overrightarrow{v_{a} v_{b}}$, then

$$
g^{v}\left(e^{\prime}\right)=\psi^{-1}\left(v_{a}\right) \tilde{h}_{v}\left(e^{\prime}\right) \psi\left(v_{b}\right)
$$

The $\psi^{\prime}$ 's are chosen so that $\left\{g^{v}\left(e^{\prime}\right)\right\}$ have the desired values on $\mathscr{E}_{I}^{r+1}$. If $v$ in $\mathscr{L}^{r+1}$ is a base point and so identified with $\hat{v}$ in $\mathscr{L}^{r}$, we require $\psi(v)=u(\hat{v})$. [Note the $g^{v}\left(e^{\prime}\right)$ may not satisfy averaging.]

Edge Smoothing. The assignments $g(e), e \in \mathscr{E}^{r+1}$ as generated so far may have undesirably large plaquette values in plaquettes at the edge of the chunk (satisfying $\left|g_{\hat{o} p}\right| \leqq c a$ only for an $N$-dependent $c$ ). We modify the assignments $g(e)$, for edges outside the chunk but within the distance $\frac{1}{4} C_{1 c} \ell_{r}$ of level $r+1$ edges of the chunk, and for edges in $E \cap \mathscr{E}_{A}^{r+1}$, so that $\left|g_{\partial p}\right| \leqq c a$ for all $S$-plaquettes (inside and outside the chunk) and averaging is satisfied. Such modification is not unique, but we make an arbitrary selection of such a smoothing. We will show in paper $V$, for suitable $c$, such smoothing is always possible for chunk fields that arise in our expansion. 


\section{Gauge Interpolation - A Herculean Task}

Field interpolation needed to be defined only in regions where the field (plaquette variables) were small. In such a region one worked in a gauge where edge assignments were locally small. In this local nearly abelian situation, the interpolation was chosen as (approximately) minimizing the (abelian) continuum action (locally). This sketchy description of the construction of the last section was given to show that there was a guiding principle that made this construction rather natural when one got under its superficial complexity. Granted our guiding principle did not at all uniquely determine details of the procedure:

Gauge interpolation seems much more complex and arbitrary. It must be defined where the gauge fields (edge assignments) are large as well as small. And we do not have as natural a guiding principle as above. We try to make the interpolated (gauge) fields as smooth as possible; we allow singularities to develop only at a discrete set of points (determined by topologically impossible-to-smooth configurations). The approximate field assignments $g(e, r)$ introduced in Sect. 9 have as one purpose the determination of these point singularities.

We begin making a few convenient definitions. We have in the past often identified vertices in different $\mathscr{V}^{r}$ that occupy the same point in $R^{4}$. The height of a vertex $v$ (in any $\mathscr{V}^{r}$ ) is the highest level, $r$, for which $v \in \mathscr{V}^{r}$ (under our identifications). We now define an $r$-plane. A plane is an $r$-plane if it contains some $p \in \mathscr{P} r$ ( $p$ and the plane viewed as lying in $R^{4}$ ). A geometric object, in $R^{4}$, is of depth $r$ if it intersects some $r$-plane, and no $r^{\prime}$-plane with $r^{\prime}<r$. A geometric object is off-r if it intersects no $r$-plane, and on-r if it does. Thus an object is of depth $r$ if it is on-r but off- $r^{\prime}$ for $r^{\prime}<r$.

A gauge, $\phi(x)$, on a set $\preceq \subset R^{4}$ is defined as a mapping $\phi: x \rightarrow G, x \in \delta$. If $\phi_{1}$ and $\phi_{2}$ are two such gauges defined on $\diamond$, we set $d^{g}\left(\phi_{1}, \phi_{2}\right)$, the distance between $\phi_{1}$ and $\phi_{2}$, to be

$$
d g\left(\phi_{1}, \phi_{2}\right)=\operatorname{Inf}_{u \in G} \sup _{x \in s} d\left(u \phi_{1}(x), \phi_{2}(x)\right) .
$$

During the course of the developments of this section, there will be a number of geometric constructions whose details (or proofs) are left to Appendix $A$. Whether viewed as technical details, or as the most interesting aspects of the present chore, it makes sense to separate them from the body of this section.

We now return to the induction step of Sect. 9. We assume $g\left(e, r^{\prime}\right)$ known for $r^{\prime} \leqq r$ and $g(e)$ for $r(e) \leqq r$; we wish to find $g(e, r+1)$. For each hypercube $H_{1}$ in $\mathscr{L}^{r}$ we will construct a gauge $\phi_{i}(x)$ living on $H_{i}$. We will use the $\phi_{i}(x)$ to determine $g(e, t+1)$. In this construction we will feel free to use $\phi_{\alpha}(x)$ associated to $H_{\alpha}$ in $\mathscr{L}^{r^{\prime}}, r^{\prime}<r$, that were constructed earlier in the inductive process! We consider hypercubes of level $(r(E)-1)$ to have had the identity field $\phi(x)=\operatorname{Id}$ all $x$, as gauge.

We call a hypercube large field, if any of the plaquettes in its boundary (24 in number) are large field. We choose the gauge assigned to such a hypercube to be the restriction of the gauge assigned (at the last inductive step) to the hypercube of one larger scale containing it:

Now let $H_{i}$ be any hypercube of level $r$ (and not large field). Consider the (partial) lattice consisting of the vertices and edges of $H_{i}$ (in number $2^{4}$ and $2^{5}$ respectively). We select an arbitrary maximal tree in this lattice. We assign a gauge 
to the vertices of $H_{i}, \phi_{i}(x)$, with the property that if $e=\overrightarrow{v_{a} v_{b}}$, for $e$ in the maximal tree, then

$$
g(e)=\phi_{i}^{-1}\left(v_{a}\right) \phi_{i}\left(v_{b}\right)
$$

This condition determines the $\phi_{i}(x)$ (on the vertices) uniquely up to a change $\phi_{i}(x) \rightarrow g \phi_{i}(x)$ for some fixed $g$. We have now determined $\phi_{i}(x)$ assigned to the hypercube $H_{i}$, as restricted to the vertices of $H_{i}$. We must extend the definition throughout the (hyper-)volume of $H_{i}$.

The extension of $\phi_{i}(x)$ is inductively, from the vertices, to the edges, then to the surfaces, then to the volumes, and finally to the hypervolume. In each step (but the last) "matching" to the gauges of the neighboring hypercubes of $H_{i}$ is important. The extensions are performed simultaneously for all $H_{i}$ (level $r$, not large field). Thus we extend each $\phi_{i}(x)$ from vertices to edges at the same time, each $\phi_{i}(x)$ from edges to surfaces at the same time, etc.

Assume the depth of $H_{i}$ is $r_{d}(i) \leqq r$. Let $\phi_{i}^{d}(x)$ be the restriction of $\phi_{\alpha}(x)$ to $H_{i}$, where $H_{\alpha}$ is the level $\left(r_{d}(i)-1\right)$ hypercube containing $H_{i}$. We will write

$$
\phi_{i}(x)=\phi_{i}^{\prime}(x) \phi_{i}^{d}(x)
$$

and thus actually we need only extend $\phi_{i}^{\prime}(x)$ from the vertices to all of $H_{i}$. (In favorable circumstances the $\phi_{i}^{\prime}(x)$ will be nearly constant on the vertices, and easier to interpolate smoothly than the $\phi_{i}(x)$. We are building in a hierachy of smoothings.)

Let $e$ be an edge in $\mathscr{E}^{r}$. It will belong to a number of hypercubes (eight in number). We will only be concerned with hypercubes that are not large field - our matching requirements will never concern large field hypercubes. Let $H_{1}$ be one of these hypercubes containing $e$ of lowest depth (largest $r_{d}$ ). We let $e=\overrightarrow{v_{a} v_{b}}$, and consider $\phi_{1}^{\prime}\left(v_{a}\right)$ and $\phi_{1}^{\prime}\left(v_{b}\right)$.

Geometric Construction 1. We find an extension of $\phi_{1}^{\prime}(x),{ }^{e} \phi_{1}^{\prime}(x)$, to a mapping from $e \rightarrow G$ (a gauge on $e$ ) such that

$$
\begin{aligned}
& \text { a) }{ }^{e} \phi_{1}^{\prime}\left(v_{a}\right) \text { and }{ }^{e} \phi_{1}^{\prime}\left(v_{b}\right) \text { assume given values. } \\
& \text { b) } \Delta_{1}\left({ }^{e} \phi_{1}^{\prime}\right) \leqq c \frac{d\left(\phi_{1}^{\prime}\left(v_{b}\right), \phi_{1}^{\prime}\left(v_{a}\right)\right)}{L_{r}}
\end{aligned}
$$

where

$$
\Delta_{1}(\phi)=\operatorname{Sup}_{x, y} \frac{d(\phi(x), \phi(y))}{|x-y|} .
$$

We note that if $\phi_{1}^{\prime}\left(v_{a}\right)$ and $\phi_{1}^{\prime}\left(v_{b}\right)$ are close enough, then they may be joined by a geodesic, and $c$ picked equal 1. We now extend the gauges to $e$ of the other hypercubes containing $e$. Let $H_{2}$ be one of these.

Geometric Construction 2. We extend $\phi_{2}(x)$ from a mapping on $v_{a}$ and $v_{b}$ to a mapping from $e \rightarrow G$, such that

$$
\begin{aligned}
& \text { a) } d^{g}\left({ }^{e} \phi_{1},{ }^{e} \phi_{2}\right) \leqq c d^{g}\left(\phi_{1}, \phi_{2}\right) \\
& \text { b) } \Delta_{1}\left({ }^{e} \phi_{2}\right) \leqq c\left(\Delta_{1}\left({ }^{e} \phi_{1}\right)+\frac{d^{g}\left(\phi_{1}, \phi_{2}\right)}{L_{r}}+\Delta_{1}\left(\phi_{2}\right)\right)
\end{aligned}
$$

where ${ }^{e} \phi$ and $\Delta_{1}$ are as above. 
We now go to the inductive step from edge (or surface) to surface (or volume). We denote this as the extension from $\partial D$ to $D$. We let $H_{1}$ be the hypercube containing $\partial D$ of lowest depth. We extend $\phi_{1}^{\prime}(x)$ defined on $\partial D$ to ${ }^{e} \phi_{1}^{\prime}(x)$ defined on $D$.

Geometric Construction 3. This extension satisfies

$$
\begin{aligned}
& \text { a) }{ }^{e} \phi_{1}^{\prime}(x)=\phi_{1}^{\prime}(x), \\
& \text { b) } \Delta_{1}\left({ }^{e} \phi_{1}^{\prime}\right) \leqq c \Delta_{1}\left(\phi_{1}^{\prime}\right),
\end{aligned}
$$

(But see caution at end of this section.)

We now extend the gauges of the other hypercubes containing $\partial D$. Let $\mathrm{H}_{2}$ be one of these:

Geometric Construction 4: We extend $\phi_{2}(x)$ on $\partial D$ to ${ }^{e} \phi_{2}(x)$ on $D$, satisfying (11.5) and (11.6).

As a final extension we assume $\phi^{\prime}(x)$ defined on the boundary of hypercube $H$, i.e. on $\partial H$. We discuss extending $\phi^{\prime}(x)$ to $H$. If the mapping

$$
\phi^{\prime}: \partial H \rightarrow G
$$

is homotopically trivial we will use the same Geometric Construction 3 to accomplish an extension ${ }^{e} \phi^{\prime}$ to all of $H$ satisfying (11.7) and (11.8): In this case we modify this ${ }^{e} \phi^{\prime}(x)$ defined on $H$ to ${ }^{e s} \phi^{\prime}(x)$ defined on $H$ [this becomes the $\phi^{\prime}(x)$ of $\left.(11.3)\right] .{ }^{e s} \phi^{\prime}$ is a smoothing of ${ }^{e} \phi^{\prime}$.

Geometric Construction 5. ${ }^{\text {es }} \phi^{\prime}(x)$ satisfies

$$
\begin{aligned}
& \text { a) }{ }^{e s} \phi^{\prime}(x)={ }^{e} \phi^{\prime}(x), \quad x \in \partial H, \\
& \text { b) }\left|D^{\alpha e s} \phi^{\prime}(x)\right| \leqq c_{\alpha} \frac{1}{\left(d(x, \partial D)^{|\alpha|-1}\right.} \Delta_{1}\left({ }^{e} \phi^{\prime}\right),
\end{aligned}
$$

where the norm on derivatives in the left side of (11.11) is any reasonable $L_{\infty}$ norm.

We finally consider $\phi^{\prime}(x)$ defined on $\partial H$ of hypercube $H$ where $\phi^{\prime}$ is not a homotopically trivial map from $\partial H$ to $G$. In this case we find an extension, discontinuous at one point $x_{0} . x_{0}$ is picked as a point in $H$ with the following property:

Centering Property. With $H$ level $r$,

$$
d\left(x_{0}, c(H)\right) \leqq L_{r+1}
$$

if $H^{\prime}$ is any cube that contains $x_{0}$, with level $H^{\prime}=r^{\prime} \geqq r$, then

$$
d\left(x_{0}, c\left(H^{\prime}\right)\right) \leqq L_{r^{\prime}+1},
$$

where $c\left(H_{i}\right)$ is the center of $H_{1}$.

Geometric Construction $6 .{ }^{e} \phi^{\prime}(x)$ defined on $H-x_{0}$, as an extension of $\phi^{\prime}(x)$ defined on $\partial H$, satisfies

a) ${ }^{e} \phi^{\prime}(x)=\phi^{\prime}(x), \quad x \in \partial H$, 
b) $\left|D^{\alpha e} \phi^{\prime}(x)\right| \leqq c_{\alpha} \cdot \operatorname{Max}\left(\frac{1}{(d(x, \partial D))^{|\alpha|-1}}, \frac{1}{\left(d\left(x, x_{0}\right)\right)^{|\alpha|-1}}\right) \cdot \frac{L_{r}}{d\left(x, x_{0}\right)} \cdot \Delta_{1}\left(\phi^{\prime}\right)$

Finally we come to specifying the $g(e, r+1)$. Let $e$, level $>r$, be $\overrightarrow{v_{a} v_{b}}$ and $e \in H_{i}, H_{i}$ level $r$; then we set

$$
g(e, r+1)=\phi_{i}^{-1}\left(v_{a}\right) \phi_{i}\left(v_{b}\right),
$$

where we have had in some cases to make an arbitrary choice of $H_{i}$ for edges contained in more than one level $r$ hypercube (lying in a boundary volume).

Caution. The geometric theorems of Appendix A, require a universal bound on $\Delta_{1}$ of $\phi$ 's (as scaled to unit scale) and it is important to check our construction maintains such where we use these theorems.

\section{Appendix A. Geometric Constructions}

\section{A. Geometric Constructions 1 and 3}

These two constructions will be treated together as part of a more general construction. Let $M$ be a compact differentiable manifold (without boundary) and provided with a Riemannian metric. Let $D$ be the unit $n$-cube,

$$
D=\left\{0 \leqq x_{i} \leqq 1\right\} \text {. }
$$

Define $\Delta_{1}$ as defined after (11.4), on any mapping from a subset of $D$ into $M$.

Theorem A.1. For each constant $c_{1}$, there is a constant $c_{2}=c_{2}\left(c_{1}\right)$, such that if $f$ is any homotopically trivial mapping from $\partial D$ into $M$ satisfying

$$
\Delta_{1}(f) \leqq c_{1}
$$

there is an extension of $f, f^{e}$, mapping $D$ into $M$, satisfying

$$
\Delta_{1}\left(f^{e}\right) \leqq c_{2} \Delta_{1}(f)
$$

We do not know if the limitation in (A.1) is necessary, whether there may not be a universal $c_{2}$.

Proof. There is an $\varepsilon>0$, such that if $\Delta_{1}(f)<\varepsilon$, then the image of $f$ lies in a piece of the manifold diffeomorphic to a cube in Euclidean space. Moreover one can set things up so that the inclusions of these Euclidean cubes into $M$ have universally bounded differential, and likewise the inverse mappings have universally bounded differential. Thus if (A.2) holds for mappings into $M^{\prime}=R^{s}$ for some constant $c$ ( $s$ the dimension of $M$ ), then (A.2) will hold in the context of the theorem for some constant $c^{\prime}$, for mappings satisfying $\Delta_{1}(f) \leqq \varepsilon$.

We wish to show that if

$$
f: \partial D \rightarrow R^{s}
$$


then there is an extension $f^{e}$

satisfying

$$
f^{e}: D \rightarrow R^{s}
$$

$$
\Delta_{1}\left(f^{e}\right) \leqq c(s) \Delta_{1}(f) .
$$

Moreover if the image of $f$ lies in a cube, $f^{e}$ can be chosen so that the image of $f^{e}$ lies in the same cube.

We now prove the statement of the last paragraph. We choose axes in $R^{s}$ parallel to the edges of the cube. It is enough that we carry out the extension for each component of the mapping separately, effectively proving (A.3)-(A.5) with $s=1$. We are reduced to proving that there is a $c$ such that if

$$
f: \partial D \rightarrow[a, b],
$$

there is an extension of $f, f^{e}$

such that

$$
f^{e}: D \rightarrow[a, b],
$$

$$
\Delta_{1}\left(f^{e}\right) \leqq c \Delta_{1}(f) .
$$

In fact we can choose the $c$ of (A.8) to be 1 ! This is a well-known extension theorem.

With the considerations above for the situation with $\Delta_{1}(f)<\varepsilon$, the proof of Theorem A.1 is now reduced to proving the existence of a $c_{2}^{\prime}\left(c_{1}\right)$ so that if

$$
\Delta_{1}(f) \leqq c_{1},
$$

$f^{e}$ may be found satisfying

$$
\Delta_{1}\left(f^{e}\right) \leqq c_{2}^{\prime} .
$$

The germ of the idea that will be used to prove this, is to look at simplicial approximations, of a sort, and note that only a finite number of mappings must be considered. A bound for each of the finite set will imply (A.10).

We consider simplicial subdivisions of $\partial D$ and of $M$. We require the inclusion maps of simplices to be differentiable. For sufficiently fine subdivision - we hold these truths to be self-evident:

1) That each $f$ satisfying (A.9) may be associated to a "good" simplicial approximation $f^{a}$ [in a sense to be specified in 2)].

2) That there is a $c_{1}^{\prime \prime}$ such that there is a mapping $f_{1}^{e}$, for each $f$

with

$$
f_{1}^{e}: \partial D \times I \rightarrow M
$$

and satisfying

$$
\begin{aligned}
& f_{1}^{e}(x, 0)=f(x), \\
& f_{1}^{e}(x, 1)=f^{a}(x),
\end{aligned}
$$

$$
\Delta_{1}\left(f_{1}^{e}\right) \leqq c_{1}^{\prime \prime}
$$

$\left(c_{1}^{\prime \prime}\right.$ independent of $f$.) This map $f_{1}^{e}$ may be chosen so that $f_{1 x}^{e}(t)=f_{1}^{e}(x, t)$ is uniform motion along a geodesic. 
3) That there is a $c_{2}^{\prime \prime}$ such that there is a mapping $f_{2}^{e}$, for each $f^{a}$

with

$$
f_{2}^{e}: \partial D \times I \rightarrow M
$$

$$
f_{2}^{e}(x, 0)=f^{a}(x), \quad f_{2}^{e}(x, 1)=x_{0}(f)
$$

[i.e. $f_{2}^{e}(x, 1)$ is a trivial map]. And $f_{2}^{e}$ satisfies

$$
\Delta_{1}\left(f_{2}^{e}\right) \leqq c_{2}^{\prime \prime}
$$

( $c_{2}^{\prime \prime}$ independent of $f$ ). Remember there are only a finite number of $f^{a}$ 's.

4) That 2) and 3) imply our theorem [verify the claim in the sentence containing (A.9) and (A.10)].

\section{B. Geometric Constructions 2 and 4}

We treat these two constructions at the same time. Let $M$ be a compact differentiable manifold (without boundary) and supplied with a metric. Given two maps $g_{1}$ and $g_{2}$ into $M$ we define $d^{M}\left(g_{1}, g_{2}\right)$

$$
d^{M}\left(g_{1}, g_{2}\right)=\sup _{x} d\left(g_{1}(x), g_{2}(x)\right) .
$$

With $B$ the until ball, we have given three maps

$$
f_{1}: \partial B \rightarrow M, f_{2}: \partial B \rightarrow M, \quad f_{1}^{e}: B \rightarrow M,
$$

where $f_{1}^{e}$ is an extension of $f_{1}$.

Theorem A.2. If $d^{M}\left(f_{1}, f_{2}\right) \leqq \varepsilon_{M}$, with $\varepsilon_{M}$ an absolute constant independent of the three maps, then there is an extension $f_{2}^{e}$ of $f_{2}$ to the ball satisfying

$$
\begin{gathered}
d^{M}\left(f_{1}^{e}, f_{2}^{e}\right) \leqq c_{1} d^{M}\left(f_{1}, f_{2}\right), \\
\Delta_{1}\left(f_{2}^{e}\right) \leqq c_{2}\left[\Delta_{1}\left(f_{1}^{e}\right)+d^{M}\left(f_{1}, f_{2}\right)+\Delta_{1}\left(f_{2}\right)\right],
\end{gathered}
$$

for some absolute constants $c_{1}$ and $c_{2}$.

Proof. We construct $f_{2}^{e}$ explicitly. We let $a$ be given as

$$
a=\operatorname{Min}\left(\frac{d^{M}\left(f_{1}, f_{2}\right)}{\Delta_{1}\left(f_{1}^{e}\right)}, \frac{1}{2}\right) \text {. }
$$

We let $B$ be centered at the origin (to simplify notation). For $|x| \leqq 1-a$ we set

$$
f_{2}^{e}(x)=f_{1}^{e}\left(\frac{x}{1-a}\right) \text {. }
$$

We determine $f_{2}^{e}(x)$ for $|x| \geqq 1-a$ by requiring

$$
f_{x}(t) \equiv f_{2}^{e}(t x)
$$

for $x \in \partial B$, to be constant speed motion along the shortest geodesic in $M$ with

$$
\begin{gathered}
f_{x}(1)=f_{2}(x), \\
f_{x}(1-a)=f_{1}(x) .
\end{gathered}
$$




\section{Geometric Construction 5}

We consider a mapping $f$ of the unit ball $B$ into $M$, a compact differentiable manifold (without boundary) equipped with a metric.

Theorem A.3. There is a mapping $f^{s}: B \rightarrow M$, such that

a) $\left.f^{s}\right|_{\hat{o} B}=\left.f\right|_{\partial B}$,

b) $\left|D^{\alpha} f^{s}(x)\right| \leqq c_{\alpha} \frac{1}{(d(x, \partial B))^{|\alpha|-1}} \Delta_{1}(f)$.

Proof: We will need a smoothing function $w(x) \in C^{\infty}$, satisfying
a) $w(x) \geqq 0$,
b) $\int w(x)=1$,
c) $w(x)=0,|x| \geqq 1$.

We define

$$
w^{h}(x)=\frac{1}{h^{s}} w\left(\frac{x}{h}\right),
$$

where $s$ is the dimension of $B$.

We also need a function $d(x) \in C^{\infty}, d: B \rightarrow R^{1}$, such that

$$
\frac{1}{2} d(x, \partial B) \leqq d(x) \leqq d(x, \partial B) .
$$

We now embed $M$ in some Euclidean space $R^{t}$, and view $f$ as a map from $B$ into $R^{t}$. We define

by

$$
f_{\varepsilon}(x): B \rightarrow R^{t}
$$

$$
f_{\varepsilon}(x)=\int d y w^{\varepsilon d(x)}(x-y) f(y)
$$

(a vector-valued integral). The distance between $f_{\varepsilon}(x)$ and $M$ in $R^{t}$ can be made uniformly small

$$
d\left(f_{\varepsilon}(x), M\right) \leqq \varepsilon^{\prime} \quad \text { all } \quad x
$$

for arbitrarily small $\varepsilon^{\prime}$ if $\varepsilon$ is small enough. If $\varepsilon^{\prime}$ is sufficiently snall, the map from the image of $f_{\varepsilon}$ to $M$, given by mapping $f_{\varepsilon}(x)$ to the closest point of $M$, is a diffeomorphism. (This is the "normal projection" onto $M$.) It is thus sufficient to verify (A.26) for such an $f_{\varepsilon} . f^{s}$ will be $f_{\varepsilon}$ followed by the normal projection onto $M$. The verification of (A.26) for $f_{\varepsilon}$ is straightforward.

\section{Geometric Construction 6}

We let $f$ be a mapping on the boundary of the unit ball $B$,

$$
\begin{gathered}
B=\left\{\left|x-x_{0}\right| \leqq 1\right\}, \\
f: \partial B \rightarrow M,
\end{gathered}
$$

$M$ a compact differentiable manifold (without boundary) equipped with a metric. 
Theorem A.4. There is a mapping $f^{e s}$ on the ball minus its center

such that

$$
f^{e s}: B-x_{0} \rightarrow M
$$

a) $\left.f^{e s}\right|_{\partial B}=\left.f\right|_{\partial B}$,

b) $\left|D^{\alpha} f^{e s}\right| \leqq c_{\alpha} \frac{1}{\left(d\left(x, \partial B \cup x_{0}\right)\right)^{|\alpha|-1}} \cdot \frac{1}{\left|x-x_{0}\right|} \cdot \Delta_{1}(f)$.

Proof. We define $d^{\prime} \in C^{\infty}$,

such that

$$
d^{\prime}: B \rightarrow R^{1}
$$

$$
\frac{1}{2} d\left(x, \partial B \cup x_{0}\right) \leqq d^{\prime}(x) \leqq d\left(x, \partial B \cup x_{0}\right)
$$

and follow the construction of subsect. $C$ using

$$
f_{\varepsilon}(x)=\int d y w^{\varepsilon d^{\prime}(x)}(x-y) f\left(\frac{y-x_{0}}{\left|y-x_{0}\right|}+x_{0}\right)
$$

instead of (A.30). Notice that in this equation the argument of $f$ remains on $\partial B$. As in the above subsection, $f$ is viewed as a mapping from $\partial B$ into $R^{t}$. The theorem follows via the same route as above in a straightforward manner.

\section{References}

1. Balaban, T.: Propagators and renormalization transformations for lattice gauge theories, I and II. Commun. Math. Phys. 95, 17-40 (1984) and 96, 223-250 (1984)

2. Federbush, P.: A phase cell approach to Yang-Mills theory. I. Modes, lattice-continuum duality. Commun. Math. Phys. 107, 319-329 (1986)

3. Federbush, P., Williamson, C.: A phase cell approach to Yang-Mills theory. II. Analysis of a mode. J. Math. Phys. 28, 1416-1419 (1987)

4. Federbush, P.: A phase cell approach to Yang-Mills theory. III. Local stability, modified renormalization group transformation. Commun. Math. Phys. 110, 293-309 (1987)

5. Federbush, P.: A phase cell approach to Yang-Mills theory. VI. Non-abelian latticecontinuum duality. Ann. l'Inst. Henri Poincaré 47, 17-23 (1987)

Communicated by A. Jaffe

Received April 17, 1987; in revised form May 7, 1987 